\title{
Variación de las cuantías de acero de refuerzo de muros de concreto para las categorías de disipación de energía definidas en NSR-10*
}

\author{
Miguel Prada* \\ Julián Carrillo*** \\ César Gélvez ${ }^{* * *}$
}

Recibido: 01/03/2016 - Aceptado: 08/09/2016

DOI: 10.22395/rium.v16n30a2

\begin{abstract}
Resumen
La mayoría de ingenieros estructurales en Colombia están interesados en evaluar la variación de las cantidades de refuerzo de muros de concreto cuando estos se diseñan para cumplir con requisitos de categorías de disipación de energía superiores a las exigidas por la NSR-10. Por tanto, en este artículo se comparan y discuten las cuantías de acero de refuerzo obtenidas del diseño de muros en concreto reforzado de dos edificios para vivienda en sistema de construcción industrializado. Los dos edificios son de distinto número de pisos, diseñados con base en distintas categorías de disipación de energía, y están localizados en diferentes zonas de amenaza sísmica. Los resultados obtenidos demostraron que, para edificios de mediana altura localizados en zona de amenaza sísmica intermedia y utilizando desplazamientos de diseño cercanos a la deriva máxima permisible, se puede generar un ahorro en la cantidad de refuerzo cuando el diseño estructural se realiza utilizando los requisitos de la categoría DES en lugar de aquellos de la categoría DMO.
\end{abstract}

Palabras clave: muro de concreto, cuantía de refuerzo, elemento especial de borde

Artículo derivado de la investigación registrada con el código interno ING-IMP-2130 de la Vicerrectoría de Investigaciones de la Universidad Militar Nueva Granada.

** Ing. civil, asistente de Investigación Grupo de Investigación Estructuras y Sísmica Facultad de ingeniería, Universidad Militar Nueva Granada, Bogotá, Colombia. Dirección electrónica: asistente2.estructuras.sismica@ unimilitar.edu.co.

*** Ing. civil, Ph.D, profesor asociado, Departamento de Ingeniería Civil, Director del Grupo de Investigación Estructuras y Sísmica, Facultad de Ingeniería, Universidad Militar Nueva Granada, Bogotá, Carrera 11 No. 101-80, Colombia. Dirección electrónica:wjcarrillo@gmail.com.

***** Ing. Civil, Ms.C, Director Nacional de Diseño MARVAL S.A. Bucaramanga, Colombia. Dirección electrónica: cgelvez@marval.com.co. 


\title{
Assessment of steel reinforcement ratios of concrete walls for energy dissipation categories defined by NSR-10 code
}

\begin{abstract}
Most structural engineers in Colombia are interested in assessing the variation of reinforcement quantities of concrete walls when designed for fulfilling requirements of categories of energy dissipation, higher than those required by nsr-10. This paper compares and discusses the steel reinforcement ratios obtained from the design of reinforced concrete walls of two housing buildings having an industrialized construction system. The two buildings have different number of stories, were designed based on different categories of seismic behavior, and are located in different seismic hazard zones. Results obtained in this study demonstrated that, for high-rise building located in intermediate seismic hazard zone and using design displacements close to the allowable drift ratio, savings in the reinforcement quantities can be obtained when structural design is carried out using requirements of des category instead of those of dmo category.
\end{abstract}

Key words: concrete walls, steel reinforcement, special boundary element. 


\section{INTRODUCCIÓN}

El Reglamento de Diseño y Construcción Sismo-resistente de edificaciones que está vigente actualmente en Colombia es la NSR-10. El reglamento propone tres categorías de desempeño sísmico del sistema estructural en términos de la capacidad de disipación de energía esperada del sistema, tales como capacidad mínima (DMI), capacidad moderada (DMO) y capacidad especial de disipación de energía (DES). Estas categorías dependen del nivel de amenaza sísmica de la zona en que se proyecta construir el edificio, y establecen tanto los requisitos de detallado estructural como también el nivel de reducción de las fuerzas sísmicas de diseño. Esto último, ya que la categoría de disipación de energía permite la reducción de la magnitud de la demanda sísmica por un factor R que incluye básicamente la capacidad de ductilidad y la sobreresistencia de la estructura. El factor R depende, además, del sistema estructural y el material utilizado.

En el caso de muros de concreto reforzado, los valores del coeficiente básico de disipación de energía $\mathrm{R}_{0}$ para DMI, DMO y DES son 2.5, 4.0 y 5.0, respectivamente. La diferencia entre los valores de $\mathrm{R}_{0}$ podría sugerir que al diseñar los muros por alguna categoría superior a la exigida como mínima por el reglamento, en una determinada zona de amenaza sísmica, se obtendría una reducción en la cantidad de material de refuerzo, debido a la influencia del sismo de diseño reducido sobre las solicitaciones de los muros. No obstante, aumentar de categoría de disipación de energía implica mayores condiciones de detallado contempladas en la NSR-10. Uribe et al. [1] analizaron un edificio de 14 pisos con muros en concreto reforzado, con el fin de evaluar el comportamiento de las cantidades de refuerzo cuando se diseña por una categoría superior a la exigida por la NSR-10 en una zona. Sin embargo, en dicho estudio solo se contempló un edificio, un único perfil de suelo (suelo D), y solo se analizan las cantidades de refuerzo de los elementos especiales de borde. Uribe et al. [1] optimizan el sistema estructural para acercarlo lo máximo posible a la deriva máxima permisible, y señalan la complejidad del análisis de estas cantidades por los requisitos de detallado. Para contemplar un mayor número de variables en el estudio y analizar los diferentes escenarios para evaluar la incertidumbre de incrementar la categoría de disipación de energía del diseño, se realizó un estudio donde se cuantificaron resultados de despieces de todos los componentes del refuerzo de muros estructurales de dos edificios reales; uno de 12 pisos y uno de 5 pisos. Para representar las condiciones de construcción más ampliamente utilizadas en Colombia, se seleccionaron edificios construidos con sistemas industrializados con muros en concreto reforzado, destinados a vivienda y típicos estructuralmente. Los edificios se ubicaron en zonas de amenaza sísmica baja e intermedia, correspondientes a Barranquilla y Bogotá, respectivamente. No se incluyó 
la zona de amenaza sísmica alta porque en esta zona la norma solo permite diseñar por DES. En cada zona se escogieron los espectros de diseño que produjeran menor deriva y mayor deriva para cada edificio. En el diseño se utilizó un análisis dinámico modal espectral y se realizaron los despieces siguiendo las especificaciones de la NSR-10 y los requerimientos de la estructura. Finalmente, se evaluaron las cantidades de acero obtenidas de los despieces y se examinó la tendencia de las cantidades en términos de altura del edificio, ubicación y categoría de disipación de energía. Los resultados permitieron evaluar el ahorro de material de refuerzo en diseño DES con respecto a DMO para cuando el sistema es más alto y más exigido.

\section{PROTOTIPOS Y DEMANDA SÍSMICA}

En el estudio se evaluaron dos edificios diferenciados entre ellos por la altura: un edificio de 5 pisos (5P) y un edificio de 12 pisos (12P). Los edificios fueron seleccionados como los más representativos de una extensa base de datos de una empresa de consultoría en diseño de edificios en Colombia. Para propósitos del estudio, se supuso que los dos edificios se encuentran ubicados en dos ciudades distintas, las cuales representan dos escenarios extremos de amenaza sísmica definidos por la NSR-10. Los diseños estructurales de cada edificio se ejecutaron con base en los requisitos de las categorías de disipación de energía permitidas en cada ciudad.

Las ciudades elegidas para el estudio fueron Barranquilla (zona de amenaza sísmica baja) y Bogotá (zona de amenaza sísmica intermedia), las cuales representan extremos de amenaza sísmica. Los lineamientos para el análisis sísmico aplicado y la deriva máxima permitida fueron establecidos con base en el título A de la NSR-10 [2]. Para el caso de Barranquilla, los espectros de diseño fueron los definidos en el Título A de la NSR-10. Cuando en la ciudad existe microzonificación, como en el caso de Bogotá, los espectros de diseño se definieron según lineamientos de esta, como indica Alcaldía Mayor de Bogotá [3]. El avalúo de cargas verticales, las combinaciones de carga, y la carga de viento se encuentran lineadas por el título B de la NSR-10. Los requisitos de materiales y de cuantías de refuerzo para elementos de concreto los contempla el título C, específicamente la sección C.14, para las generalidades de muros, y la sección C.21, para los elementos que componen el sistema sismo-resistente, donde se indican requisitos especiales según cada categoría de disipación de energía y tipo de elemento.

\subsection{Descripción de los edificios}

Los edificios de este estudio están conformados por muros sin cambios significativos de rigidez en su altura, sin aberturas en ellos, con espesores que varían entre $120 \mathrm{~mm}$ y 
150 mm, y muros de sección recta; es decir, que para la flexo-compresión no se tendrá en cuenta el aporte de las aletas formadas por la intersección de dos muros. La altura libre de entrepiso de los dos edificios es de $2.35 \mathrm{~m}$ y el espesor de la losa maciza de entrepiso es de $100 \mathrm{~mm}$. Los sistemas industrializados suelen generar una composición estructural tal que en una dirección permita separaciones que varían entre $2.5 \mathrm{~m}$ y $3.5 \mathrm{~m}$, con el objetivo de utilizar losas de entrepiso con espesores que varían entre 100 $\mathrm{mm}$ y $120 \mathrm{~mm}$, y que funcionen con refuerzo mínimo, señalan Mayagoitia et al. [4]. Esto implica que en uno de los sentidos de la planta se tendrá mayor densidad de muros en comparación con la dirección ortogonal. En la figura 1 se muestra un esquema de la planta de cada edificio. En la tabla 1 se muestra un resumen de las características de los edificios estudiados. En la tabla, la densidad de muros, evaluada en cada dirección, corresponde a la relación entre el área total de muros por planta y el área de la planta. El $f_{c}^{\prime}$ usado en cada edificio corresponde a valores típicos para la altura de cada edificio, debido a que este es un parámetro que influye en todo el diseño estructural.
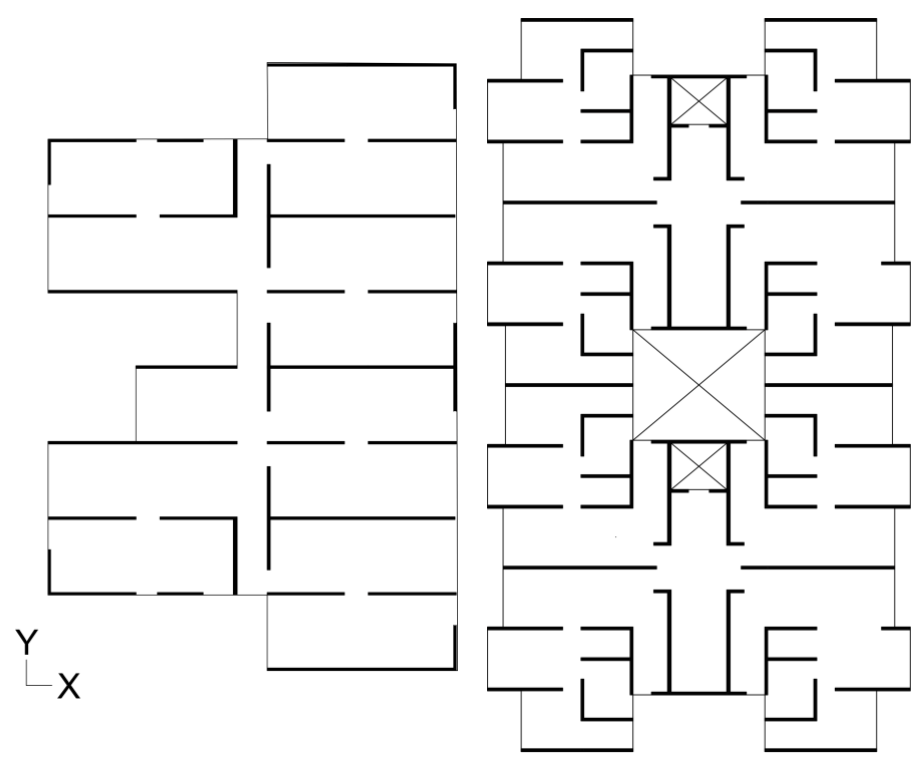

Figura 1. Configuración en planta de edificios: (a) 5P, (b) 12P. Fuente: elaboración propia 
Tabla 1. Características principales de los edificios estudiados

\begin{tabular}{|c|c|c|c|}
\hline \multicolumn{2}{|r|}{ Parámetro } & $5 P$ & $12 P$ \\
\hline \multicolumn{2}{|l|}{ Número de pisos } & 5 & 12 \\
\hline \multicolumn{2}{|c|}{ Altura libre de entrepiso, $\mathrm{m}$} & 2.35 & 2.35 \\
\hline \multicolumn{2}{|c|}{ Altura total del edificio, m } & 12.3 & 29.4 \\
\hline \multicolumn{2}{|c|}{ Resistencia a compresión del concreto, $f_{c}^{\prime}, \mathrm{MPa}$} & 21 & 28 \\
\hline \multicolumn{2}{|c|}{ Esfuerzo de fluencia del acero de refuerzo, $f_{y}, \mathrm{MPa}$} & 420 & 420 \\
\hline \multirow{2}{*}{ Densidad de muros, $\%$} & Eje X & 3.6 & 4.9 \\
\hline & Eje Y & 1.2 & 2.1 \\
\hline \multirow{4}{*}{ Carga viva $\mathrm{kN} / \mathrm{m}^{2}$} & Entrepiso & 1.8 & 1.8 \\
\hline & Azotea & 0.5 & 0.5 \\
\hline & Escalera & 3.0 & 3.0 \\
\hline & Cuarto de máquinas & Sin cuarto & 0.5 \\
\hline \multirow{4}{*}{ Carga muerta $\mathrm{kN} / \mathrm{m}^{2}$} & Entrepiso & 5.1 & 5.7 \\
\hline & Azotea & 5.0 & 4.0 \\
\hline & Escalera & 17.0 & 17.0 \\
\hline & Cuarto de máquinas & Sin cuarto & 10.0 \\
\hline \multicolumn{2}{|l|}{ Peso del edificio, $\mathrm{kN}$} & 12700 & 60400 \\
\hline \multirow{2}{*}{ Periodo de vibración, $\mathrm{s}$} & Eje $X$ & 0.13 & 0.81 \\
\hline & Eje Y & 0.40 & 0.98 \\
\hline \multirow{2}{*}{$\begin{array}{l}\text { Periodo de vibración } \\
\text { aproximado, s }\end{array}$} & Eje $X$ & 0.09 & 0.50 \\
\hline & Eje Y & 0.25 & 1.10 \\
\hline
\end{tabular}

Fuente: elaboración propia

\subsection{Demanda sísmica y análisis dinámico}

El mapa de zonificación sísmica de Colombia divide el territorio en tres grandes zonas de amenaza sísmica: baja, intermedia y alta. La NSR-10 establece una categoría mínima de disipación de energía que se debe utilizar para el diseño en cada zona de amenaza sísmica, tal como se muestra en la tabla 2. Para este estudio, las dos ciudades elegidas fueron Barranquilla (zona de amenaza sísmica baja) y Bogotá (zona de amenaza sísmica intermedia). Para el caso de Bogotá, la amenaza sísmica se define en la microzonificación sísmica de la ciudad. 
Tabla 2. Zonas de amenaza sísmica

y categorías de disipación de energía permitidas en NSR-10 para diseño

\begin{tabular}{|c|c|c|c|}
\hline $\begin{array}{c}\text { Zona de amenaza } \\
\text { sísmica }\end{array}$ & $\begin{array}{c}\text { ¿Se puede diseñar por } \\
\text { DMI? }\end{array}$ & $\begin{array}{c}\text { ¿Se puede diseñar por } \\
\text { DMO? }\end{array}$ & $\begin{array}{c}\text { ¿Se puede diseñar por } \\
\text { DES? }\end{array}$ \\
\hline Baja & Sí & Sí & Sí \\
\hline Intermedia & No & Si & Sí \\
\hline Alta & No & No & Sí \\
\hline
\end{tabular}

Fuente: elaboración propia

Para seleccionar los tipos de suelo que se utilizarían en el estudio, los edificios se modelaron y analizaron para todos los posibles espectros de cada zona (diferentes tipos de suelo). Para cada edificio, en cada zona de amenaza sísmica (baja e intermedia), se escogieron los espectros que generaron menor deriva de piso y mayor deriva de piso sin exceder la máxima permisible. De acuerdo con Carrillo et al. (2013) [5], el límite de deriva establecido por la NSR-10 está más relacionado con un indicador de desempeño o límite de control relacionado con el estado límite de servicio. Para estructuras en mampostería estructural, este límite es de $0.5 \%$ por la naturaleza frágil de los elementos en este material, y para las demás estructuras es de $1.0 \%$. Cuando en el análisis estructural se usan secciones fisuradas, los resultados de la deriva obtenidos en el análisis se deben reducir a 70\%, antes de compararlos con los límites establecidos por la NSR-10. Sin embargo, en los análisis estructurales de este estudio no se usaron secciones fisuradas. Los espectros de diseño elegidos para cada edificio se enlistan en la tabla 3 , y se muestran en las figuras 2 y 3 , respectivamente.

Tabla 3. Espectros de diseño para los casos estudiados

\begin{tabular}{|c|c|c|c|c|c|}
\hline \multicolumn{2}{|c|}{ Ubicación } & \multicolumn{2}{c|}{$5 P$} & \multicolumn{2}{c|}{$12 P$} \\
\cline { 3 - 6 } & \multicolumn{2}{c|}{ Deriva del sistema } & \multicolumn{2}{c|}{ Deriva del sistema } \\
\hline $\begin{array}{c}\text { Zona de amenaza } \\
\text { sísmica }\end{array}$ & Ciudad & $\begin{array}{c}\text { Mínima } \\
(\text { MIN) }\end{array}$ & $\begin{array}{c}\text { Máxima } \\
\text { (MAX) }\end{array}$ & $\begin{array}{c}\text { Mínima } \\
\text { (MIN) }\end{array}$ & $\begin{array}{c}\text { Máxima } \\
\text { (MAX) }\end{array}$ \\
\hline Intermedia & Bogotá & Lacustre-500 & Piedemonte B & Cerros & Lacustre-200 \\
\hline Baja & Barranquilla & Suelo A & Suelo E & Suelo A & Suelo D \\
\hline
\end{tabular}

Fuente: elaboración propia

Para el diseño de los edificios se utilizó un análisis dinámico modal espectral. El período fundamental de vibración de los dos edificios, en cada dirección, se muestra en la tabla 1. Para verificar el requisito de cortante basal mínimo, en la tabla se incluye el período de vibración aproximado de la estructura. Este período aproximado se calcula 
con la ecuación propuesta por la NSR-10, en la cual se considera las dimensiones y alturas de los muros del edificio.
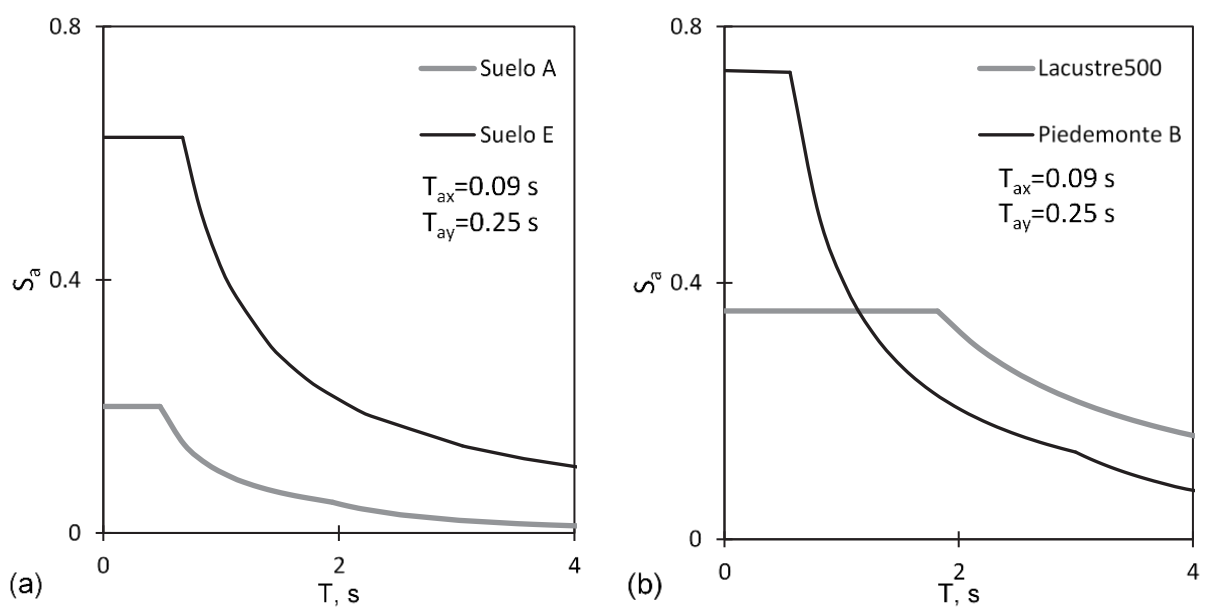

Figura 2. Espectros de diseño edificio 5P: (a) Barranquilla y (b) Bogotá.

Fuente: elaboración propia
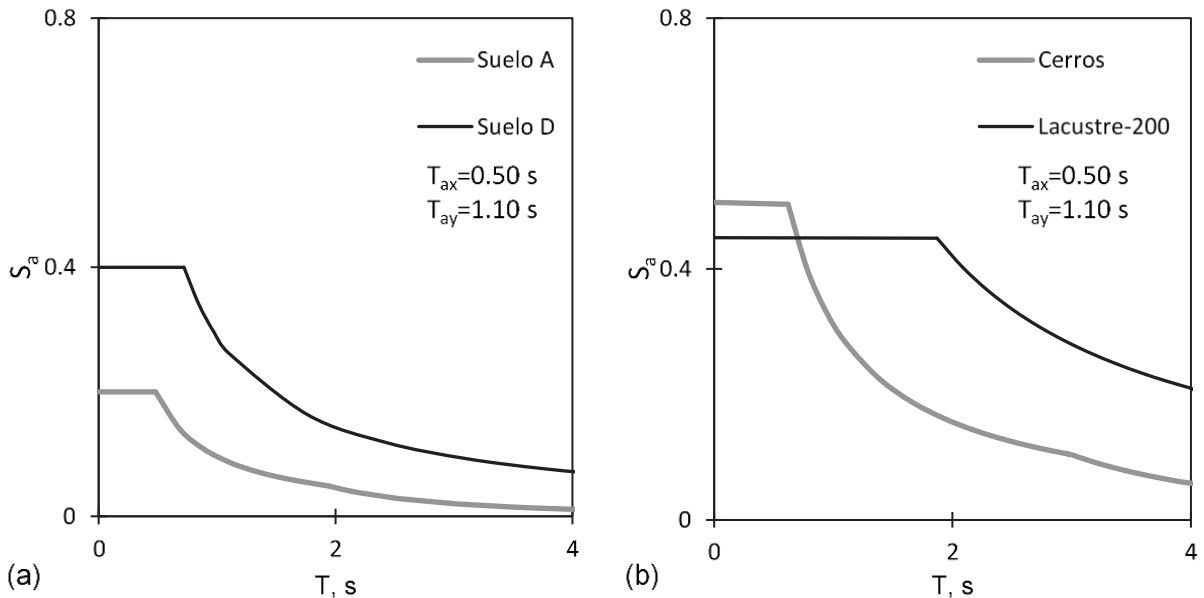

Figura 3. Espectros de diseño edificio 12P: (a) Barranquilla y (b) Bogotá. Fuente: elaboración propia 


\section{PARÁMETROS PARA DISEÑO DE MUROS}

La sección C.21 de la NSR-10 [2] contempla los requisitos de refuerzo para los elementos sismo-resistentes. En general, a medida que aumenta la categoría de disipación de energía, se incrementan los requisitos de confinamiento del muro para ofrecer ductilidad y, por tanto, se requieren elementos especiales de borde para los muros diseñados en DMO y DES; estos elementos no son requeridos en DMI. El refuerzo del muro se divide básicamente en dos partes principales: refuerzo del alma y refuerzo en los bordes. El refuerzo en el alma busca satisfacer la resistencia a cortante y la transferencia de cortante. El refuerzo en los bordes responde a requerimientos de suministro de ductilidad al muro, y puede también ser requerido para satisfacer las demandas de flexo-compresión.

\subsection{Transferencia de cortante}

Dado que el sistema constructivo contempla fundir monolíticamente cada piso, el concreto ya endurecido de un piso fundido forma una junta fría con el concreto recién fundido del nuevo piso. Para que el muro se comporte monolíticamente, se debe transmitir el cortante entre las dos caras de la junta. El mecanismo de transmisión se conoce como "transmisión por fricción", ya que se toma en cuenta la fricción entre las dos caras de la junta para estimar un área de refuerzo según McCormac y Nelson [6], la cual se suple mediante la cuantía longitudinal del alma a cortante. La figura 4 muestra la sección de un muro de longitud $L_{w}$, con recubrimiento del refuerzo $2 \mathrm{~cm}$, dividida en $n$ partes, según el diámetro $d_{b}$ y la separación $s$ del refuerzo longitudinal.

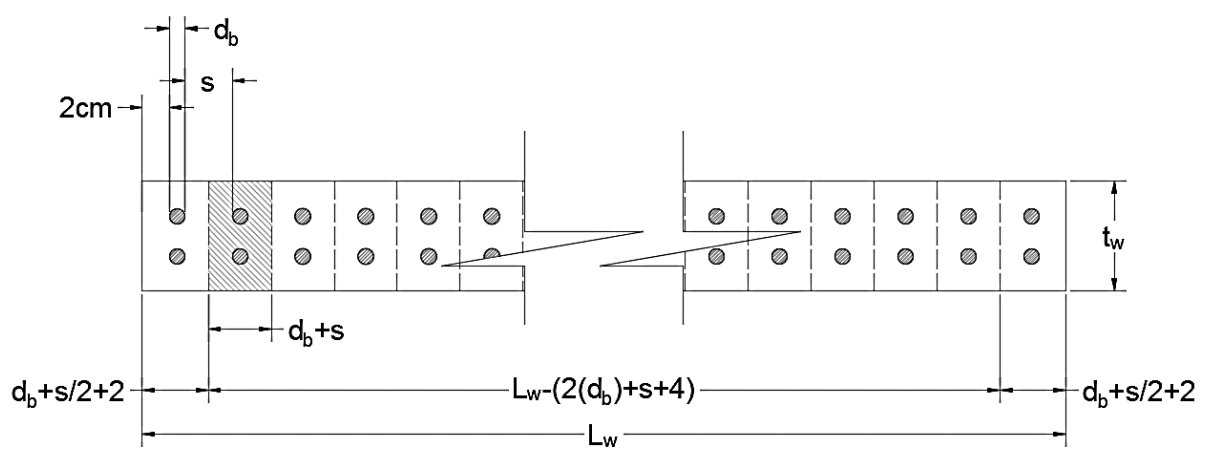

Figura 4. Esquema de "bloques" de la sección del muro.

Fuente: elaboración propia 
En la tabla 4, $n$ representa la cantidad de segmentos o "bloques" cuyas dimensiones son el espesor del muro $t_{w}, \mathrm{y} d_{b}+s$. En cada segmento actúa una fuerza cortante aferente $\tilde{V}_{u}$, relacionada con la fuerza cortante que actúa sobre el muro en la zona donde se va a formar la junta $V_{u}$, obtenido del análisis estructural. El valor del cortante aferente se encuentra limitado con los valores de las ecuaciones que se muestran en la tabla 4. En la tabla, $A_{v l}$ es el área de refuerzo requerida por cada cortina de refuerzo $n_{c o r}$, en cada uno de los bloques. Cuando esta área es menor o igual al área del refuerzo longitudinal, se cumple con la transferencia de cortante.

Tabla 4. Expresiones usadas para calcular de transferencia de cortante por fricción

\begin{tabular}{|c|c|c|}
\hline & $n=\frac{L_{w}-\left(\mathrm{s}+d_{b}\right)-0.04}{s+\mathrm{d}_{b}}$ & $\tilde{V} u=\frac{V_{u}}{n}(k N)$ \\
\hline$\tilde{V} u \leq$ & 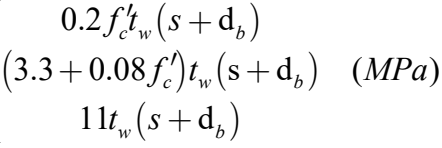 & $A_{v l}=\frac{\tilde{V} u}{6 \mathrm{~d}_{b} f_{y} n_{c o r}}\left(\mathrm{~mm}^{2}\right)$ \\
\hline
\end{tabular}

Fuente: elaboración propia

\subsection{Elementos especiales de borde}

Como se indicó en la sección 2, los elementos especiales de borde aplican en el diseño de muros con DMO y con DES. La NSR-10 tiene dos criterios que definen cuándo se requieren elementos especiales de borde. Un criterio adoptado por desempeño se basa en el desplazamiento del último nivel de cada muro. El segundo criterio relaciona el esfuerzo a compresión en la sección transversal del muro con un porcentaje de $f_{c}^{\prime}$ para decidir si se requiere un elemento especial de borde. En la tabla 5 se definen los valores para este segundo criterio. Para facilitar el proceso de diseño, en este estudio se adoptó el segundo criterio. Entre la opción de "requerir" y "no requerir" elementos de borde, la norma deja un intervalo de $(15 \%, 20 \%)$ para DES y $(22 \%, 33 \%)$ para DMO sobre el cual no especifica. Por tanto, en este trabajo se decidió que solo cuando se cumple el porcentaje para "no requerir", se elimina el elemento especial de borde.

Tabla 5. Criterio de condicionamiento basado en el esfuerzo a compresión del concreto para elementos especiales de borde

\begin{tabular}{|c|c|c|}
\hline \multirow{2}{*}{ Categoria } & \multicolumn{2}{|c|}{ Porcentaje delf'c } \\
\cline { 2 - 3 } & Para requerir & Para no requerir \\
\hline DES & $\geq 20$ & $\leq 15$ \\
\hline DMO & $\geq 33$ & $\leq 22$ \\
\hline
\end{tabular}


Como se muestra en la figura 5, el muro en un piso $i$ es sometido a un momento $M_{u i}$, una carga axial de compresión $P_{u i}$ y un cortante solicitante $V_{u i}$ El momento se puede descomponer en un sistema de pares que actúa como fuerza axial en el centroide de cada elemento especial de borde del piso (achurado en la figura 5), el cual tiene un ancho $C_{b i}$ relacionado con la profundidad de compresión del muro.

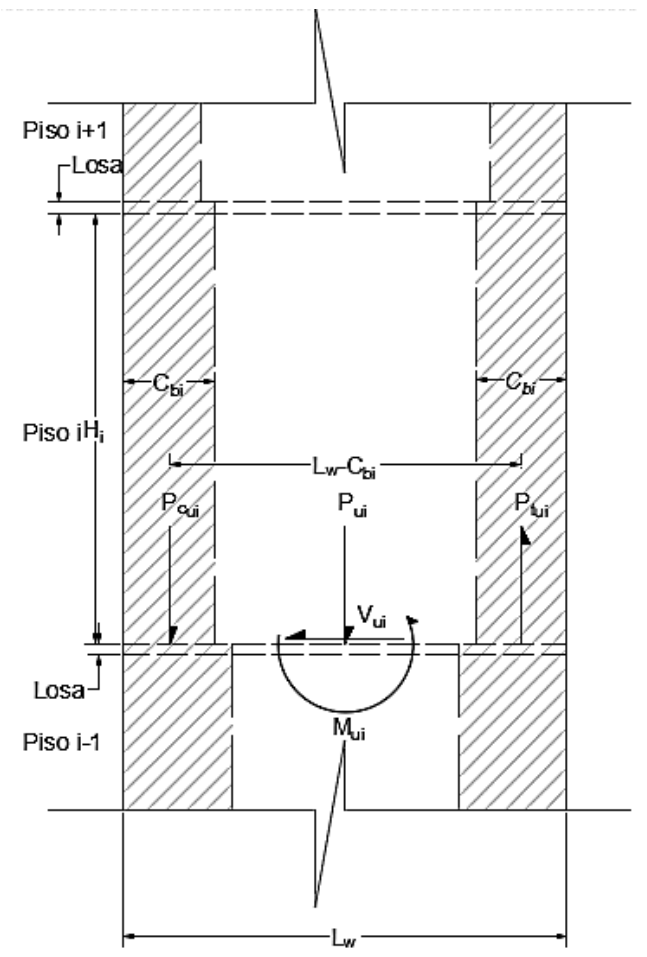

Figura 5. Diagrama de cuerpo libre del muro. Fuente: elaboración propia

La naturaleza de los elementos especiales de borde es similar a la función de una columna Mohele [7]. Estos elementos se diseñan con un refuerzo longitudinal para satisfacer el par de fuerzas en que se descompone el momento. Este refuerzo, en su mayor parte, responde a solicitaciones de compresión. Adicionalmente, se utilizan estribos de confinamiento en toda su altura para configurar suficiente ductilidad al muro y controlar el pandeo local de las barras longitudinales como han mencionado Hube, Alarcon y Massone [8-10]. 


\section{RESULTADOS Y DISCUSIÓN}

Luego de analizar, diseñar y detallar el acero de refuerzo de los muros de cada edificio, se calcularon las cantidades totales de refuerzo en cada uno de los casos del estudio. Para facilitar la discusión de los resultados, la nomenclatura utilizada en cada caso es la siguiente:

$$
B q \underbrace{05}_{2} \underbrace{M x}_{3} \underbrace{1}_{4}
$$

donde 1 indica la ciudad en que se ubicó el edificio, Barranquilla $(\mathrm{Bq})$ o Bogotá (Bt); 2 indica el edificio, 5P(05) o 12P(12); 3 indica el espectro relacionado con la máxima (MAX) o mínima (MIN) deriva (según tablas 2 y 3), y 4 indica la categoría de disipación de energía que se utilizó en el diseño DMI(1), DMO(2) y DES(3). En el estudio se evaluaron las cantidades en los componentes principales del refuerzo de los muros: el refuerzo en el alma, MA; el refuerzo longitudinal de los elementos de borde, LB, y el refuerzo usado para confinar los elementos especiales de borde, CB. En este estudio, el refuerzo en el alma de los muros se suplió usando mallas electrosoldadas. Las figuras 6 a 9 muestran los resultados de estas cantidades.
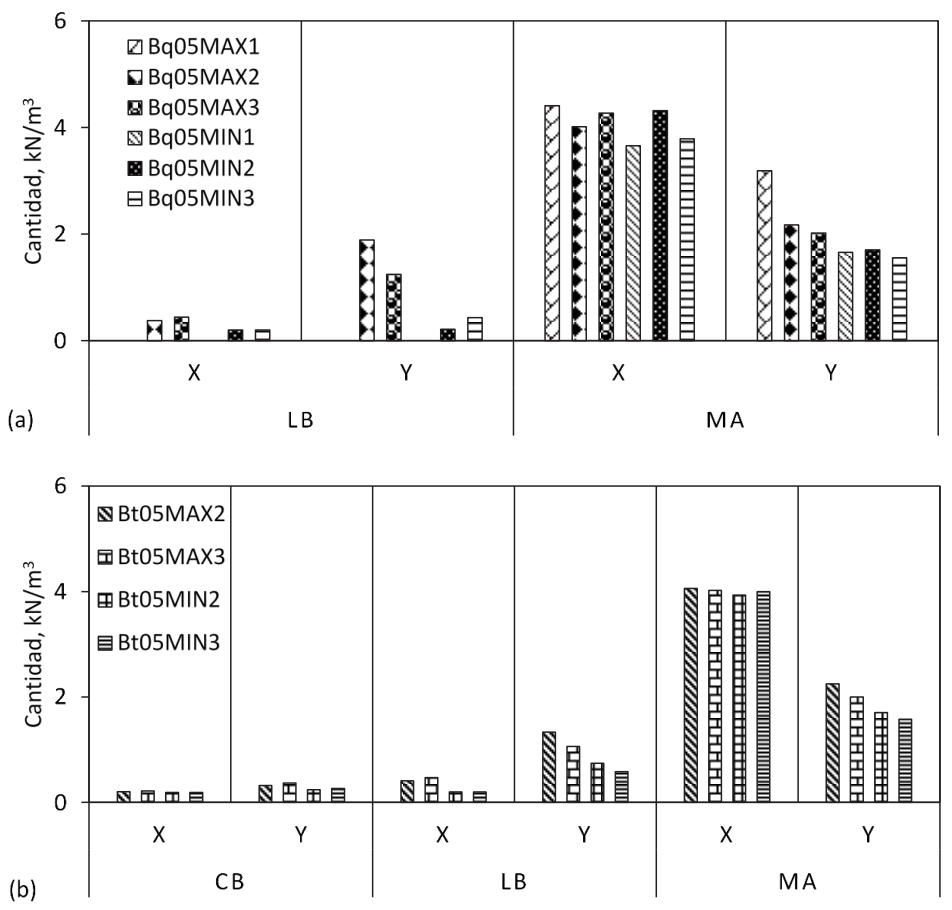

Figura 6. Cantidades de refuerzo en cada dirección del edificio 5P: (a) Barranquilla (b) Bogotá. Fuente: elaboración propia 

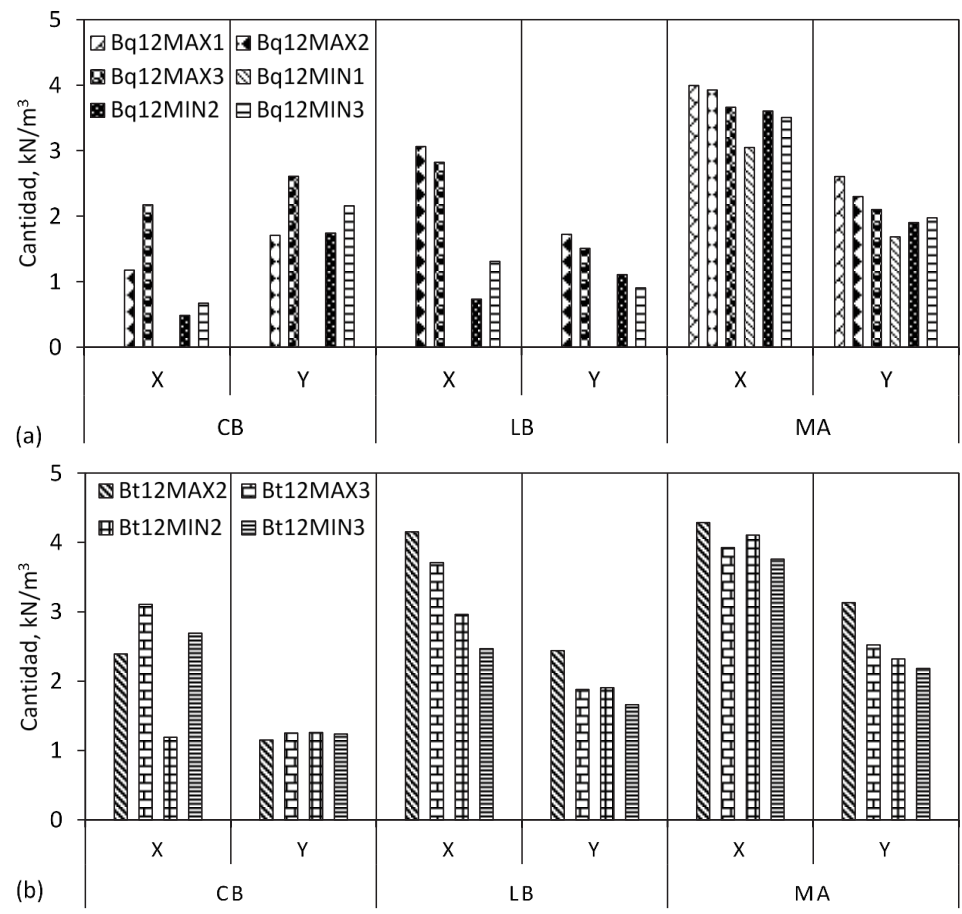

Figura 7. Cantidades de refuerzo en cada dirección 12P (a) Barranquilla (b) Bogotá. Fuente: elaboración propia
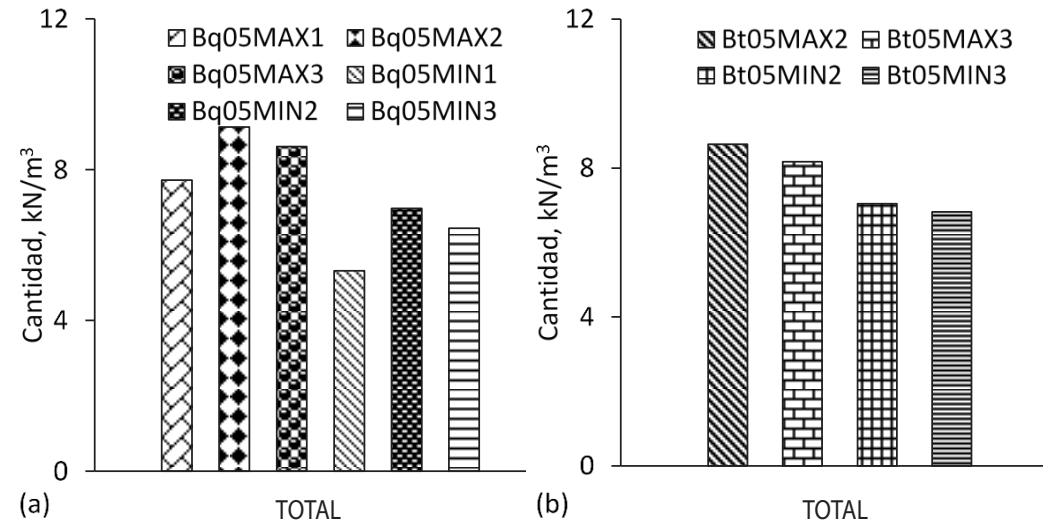

Figura 8. Cantidades totales de refuerzo del edificio 5P: (a) Barranquilla (b) Bogotá. Fuente: elaboración propia 

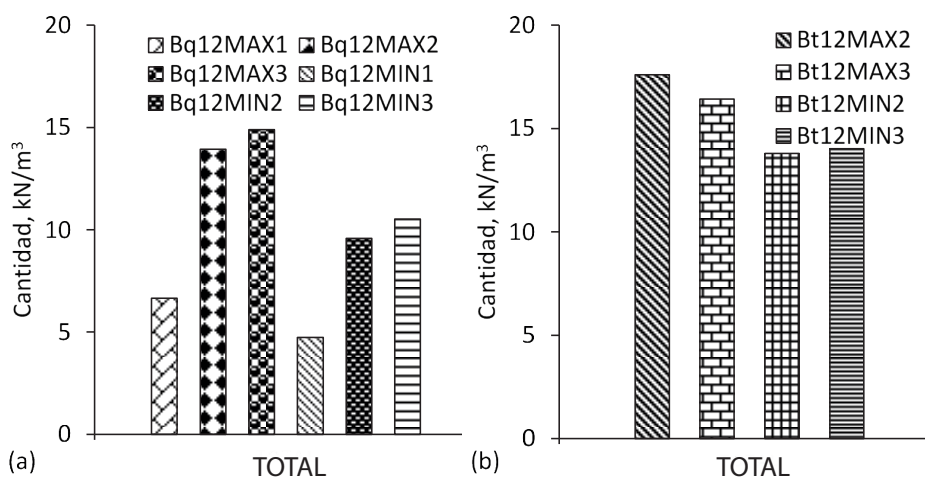

Figura 9. Cantidades s de refuerzo total 12P (a) Barranquilla (b) Bogotá. Fuente: elaboración propia

En figura 6 se observa que el componente del refuerzo que domina en el diseño de los muros en edificios de baja altura es el refuerzo en el alma MA. El comportamiento del componente MA en Barranquilla (figura 6a), a medida que la estructura se aleja de la deriva máxima permisible $(1.0 \%)$, en la dirección con mayor densidad de muros se requiere menor cantidad de refuerzo para un diseño con DMI $\left(3.8 \mathrm{kN} / \mathrm{m}^{3}\right)$ comparado con un diseño con DMO $\left(4.3 \mathrm{kN} / \mathrm{m}^{3}\right)$ y, prácticamente, la misma cantidad que para un diseño DES $\left(3.7 \mathrm{kN} / \mathrm{m}^{3}\right)$. Sin embargo, al acercarse a la máxima deriva permisible, se requiere más cantidad de refuerzo para un diseño DMI $\left(4.3 \mathrm{kN} / \mathrm{m}^{3}\right)$ que para un diseño DMO $\left(4.0 \mathrm{kN} / \mathrm{m}^{3}\right)$, y la misma cantidad comparada con un diseño DES $\left(4.4 \mathrm{kN} / \mathrm{m}^{3}\right)$. En la dirección de menor densidad de muros, al alejarse de la máxima deriva permisible, DES, DMO y DMI requieren prácticamente la misma cantidad de refuerzo $\left(1.7 \mathrm{kN} / \mathrm{m}^{3}, 1.7 \mathrm{kN} / \mathrm{m}^{3}\right.$ y $1.6 \mathrm{kN} / \mathrm{m}^{3}$, respectivamente). Al acercarse a la deriva máxima permisible, DES $\left(3.2 \mathrm{kN} / \mathrm{m}^{3}\right)$ requiere mayor cantidad de refuerzo comparado con DMI $\left(2.0 \mathrm{kN} / \mathrm{m}^{3}\right)$ y prácticamente la misma cantidad que DMO $\left(2.2 \mathrm{kN} / \mathrm{m}^{3}\right)$. En Bogotá (figura 6b), en la dirección de mayor densidad de muros, el componente MA permanece prácticamente constante entre DES y DMO, independientemente de la deriva del sistema. En la dirección de menor densidad de muros, el diseño por DES $\left(2.2 \mathrm{kN} / \mathrm{m}^{3}\right)$ requiere más cantidad de refuerzo que DMO $\left(2.0 \mathrm{kN} / \mathrm{m}^{3}\right)$, a medida que la deriva del sistema se acerca a la máxima permisible; en el caso de mínima deriva, ambos diseños (DMO y DES) requieren la misma cantidad de refuerzo $\left(1.7 \mathrm{kN} / \mathrm{m}^{3} \mathrm{y}\right.$ $1.6 \mathrm{kN} / \mathrm{m}^{3}$, respectivamente).

El componente LB en Barranquilla (figura 6a) en la dirección de mayor densidad de muros permanece prácticamente igual entre los diseños DMO $\left(0.4 \mathrm{kN} / \mathrm{m}^{3}\right.$ máxima deriva y $0.2 \mathrm{kN} / \mathrm{m}^{3}$ mínima deriva) y DES $\left(0.4 \mathrm{kN} / \mathrm{m}^{3}\right.$ máxima deriva y 0.2 
$\mathrm{kN} / \mathrm{m}^{3}$ mínima deriva), independientemente de la deriva. En cambio, se observa que en la dirección de menor densidad de muros el diseño DES requiere menor cantidad de refuerzo en este componente a medida que la deriva del sistema se acerca a la máxima permisible; por ejemplo, en el caso de mínima deriva, $0.2 \mathrm{kN} / \mathrm{m}^{3}$ para DES y $0.4 \mathrm{kN} / \mathrm{m}^{3}$ para DMO; y en el caso de máxima deriva, $1.2 \mathrm{kN} / \mathrm{m}^{3}$ para DES y $1.9 \mathrm{kN} /$ $\mathrm{m}^{3}$ para DMO. En Bogotá (figura 6b), en la dirección de menor densidad de muros, para un diseño DES, el componente LB requiere menor cantidad de refuerzo que el diseño DMO; esta tendencia se mantiene independiente de la deriva del sistema. En la dirección de mayor densidad de muros, el diseño DES requiere prácticamente la misma cantidad de refuerzo que el diseño DMO, independientemente de la deriva del sistema. El componente $\mathrm{CB}$ permanece igual e indiferente a la deriva del sistema para el diseño DMO y DES, en ambas ciudades.

En la figura 7 se muestra que el componente de refuerzo MA pierde el protagonismo que tenía en el caso del edificio 5P, en términos de las cantidades de refuerzo. En Barranquilla (figura 7a) el componente MA, en la dirección de mayor densidad de muros, se observa que el componente MA entre DMO $\left(3.6 \mathrm{kN} / \mathrm{m}^{3}\right)$ y DES $\left(3.5 \mathrm{kN} / \mathrm{m}^{3}\right)$, requiere prácticamente la misma cantidad de refuerzo, y DES $(3.5 \mathrm{kN} /$ $\left.\mathrm{m}^{3}\right)$ requiere más que DMI $\left(3.0 \mathrm{kN} / \mathrm{m}^{3}\right)$ en el caso de mínima deriva. Al acercarse a la máxima deriva permisible $(1.0 \%), \mathrm{DMO}\left(3.9 \mathrm{kN} / \mathrm{m}^{3}\right)$ requiere prácticamente la misma cantidad de refuerzo que DMI $\left(4.0 \mathrm{kN} / \mathrm{m}^{3}\right)$; sin embargo, DES $\left(3.7 \mathrm{kN} / \mathrm{m}^{3}\right)$ requiere menor cantidad de refuerzo que DMI. En la dirección de menor densidad de muros se observa un comportamiento similar; por ejemplo, para el caso de mínima deriva, DMI $\left(1.7 \mathrm{kN} / \mathrm{m}^{3}\right)$ requiere menor cantidad de refuerzo que DES $\left(2.0 \mathrm{kN} / \mathrm{m}^{3}\right)$ y DMO $\left(1.9 \mathrm{kN} / \mathrm{m}^{3}\right)$, y al acercarse el sistema a la máxima deriva permisible, DMI $\left(2.6 \mathrm{kN} / \mathrm{m}^{3}\right)$ requiere más material de refuerzo que DMO $\left(2.3 \mathrm{kN} / \mathrm{m}^{3}\right)$ y aún más que DES $\left(2.1 \mathrm{kN} / \mathrm{m}^{3}\right)$. En Bogotá (figura $7 \mathrm{~b}$ ) se observa que el componente MA del refuerzo sigue teniendo una participación importante en las cantidades de refuerzo, y con respecto a Barranquilla genera mayores valores en ambas direcciones y para ambos casos de deriva (máxima y mínima). Se observa también en la figura $7 \mathrm{~b}$ que, independientemente de la dirección y de la deriva del sistema, DMO requiere mayor cantidad de refuerzo para este componente que DES; por ejemplo, para la dirección de mayor densidad de muros en el caso de máxima deriva, DMO requirió $4.3 \mathrm{kN} / \mathrm{m}^{3}$ y DES requirió $3.9 \mathrm{kN} / \mathrm{m}^{3}$. Sin embargo, a medida que el sistema se acerca a la máxima deriva permisible, la relación entre la cantidad de refuerzo en el componente MA para el diseño DES y para el diseño DMO (DES/DMO) indica que tanto para la dirección de mayor densidad de muros $(\mathrm{DES} / \mathrm{DMO}=0.90)$ como para la dirección de menor densidad de muros $(\mathrm{DES} / \mathrm{DMO}=0.81)$, se logra requerir menor cantidad de refuerzo, que al alejar el sistema de la máxima deriva permisible $(\mathrm{DES} / \mathrm{DMO}=0.92$ dirección de mayor densidad 
de muros y $\mathrm{DES} / \mathrm{DMO}=0.96$ dirección de menor densidad de muros).

En Barranquilla (figura 7a), el componen LB del refuerzo en la dirección de mayor densidad de muros se observa que, al alejarse de la deriva máxima permisible, el diseño por DMO $\left(0.7 \mathrm{kN} / \mathrm{m}^{3}\right)$ requiere menor cantidad de refuerzo que DES $\left(1.3 \mathrm{kN} / \mathrm{m}^{3}\right)$ y al acercarse el sistema a la máxima deriva permisible, DES $\left(2.8 \mathrm{kN} / \mathrm{m}^{3}\right) \operatorname{logra}$ requerir menor cantidad de refuerzo que DMO $\left(3.1 \mathrm{kN} / \mathrm{m}^{3}\right)$. En la dirección de menor densidad de muros, independientemente de la deriva del sistema, DMO requiere más cantidad de refuerzo que DES; por ejemplo, para el caso de máxima deriva, DMO requirió $1.7 \mathrm{kN} / \mathrm{m}^{3}$ y DES $1.5 \mathrm{kN} / \mathrm{m}^{3}$. Para el edificio 12P. En Bogotá (figura $7 \mathrm{~b}$ ) el componente LB, independientemente de la dirección y de la deriva del sistema DMO, requiere mayor cantidad de refuerzo que DES; por ejemplo, para la dirección de mayor densidad de muros en el caso de máxima deriva, DMO requirió $4.2 \mathrm{kN} / \mathrm{m}^{3}$ y DES requirió $3.7 \mathrm{kN} / \mathrm{m}^{3}$. Sin embargo, la relación entre la cantidad de refuerzo en este componente para DES y para DMO en la dirección de mayor densidad de muros sugiere que se requiere menor cantidad de refuerzo para DES a medida que el sistema se aleja de la deriva máxima permisible; esto es, $\mathrm{DES} / \mathrm{DMO}=0.83$ para el caso de mínima deriva y $\mathrm{DES} / \mathrm{DMO}=0.88$ para el caso de máxima deriva. $\mathrm{El}$ caso contrario ocurre en la dirección de menor densidad de muros en la que $\mathrm{DES} / \mathrm{DMO}=0.79$, para el caso de máxima deriva, y $\mathrm{DES} / \mathrm{DMO}=0.89$, para el caso de mínima deriva.

En Barranquilla (figura 7a), el componente CB logra mayor participación en comparación con el edificio 5P, y se observa que independientemente de la dirección y de la deriva del sistema, DMO requiere menor cantidad de refuerzo que DES; por ejemplo, para la dirección de mayor densidad de muros en el caso de máxima deriva, DES requirió $2.2 \mathrm{kN} / \mathrm{m}^{3}$ y DMO $1.2 \mathrm{kN} / \mathrm{m}^{3}$. En Bogotá (figura $7 \mathrm{~b}$ ) el componente CB en la dirección de menor densidad de muros, la cantidad de refuerzo que se requiere es prácticamente la misma para ambos caos DES $\left(1.3 \mathrm{kN} / \mathrm{m}^{3}\right.$ para máxima deriva y $1.2 \mathrm{kN} / \mathrm{m}^{3}$ mínima deriva) y DMO $\left(1.2 \mathrm{kN} / \mathrm{m}^{3}\right.$ para máxima deriva y $1.3 \mathrm{kN} / \mathrm{m}^{3}$ para mínima deriva). En la dirección de mayor densidad de muros, DMO requiere siempre menor cantidad de refuerzo que DES, y menor cantidad en la medida que el sistema se aleja de la deriva máxima permisible; por ejemplo, para el caso de máxima deriva, $\mathrm{DMO} / \mathrm{DES}=0.77$ y para el caso de mínima deriva, $\mathrm{DMO} / \mathrm{DES}=0.44$.

Las figuras 8 y 9 muestran las cantidades totales de refuerzo de cada caso de estudio para los dos edificios. El edificio 5P localizado en Barranquilla (figura 8a) muestra claramente que la cantidad de refuerzo en diseño con DMI es siempre menor que la cantidad requerida por un diseño con DMO o un diseño con DES. Cuando se comparan los resultados del diseño con DMO y el diseño con DES, este último requiere en total menor cantidad de refuerzo que DMO en el caso de mínima deriva; por ejemplo, la 
relación entre la cantidad de material para el diseño DMI y el diseño DES es de 0.8 y 0.9 para los casos de mínima deriva y de máxima deriva, respectivamente. En este mismo edificio localizado en Bogotá (figura 8b), el diseño por DMO requiere en total prácticamente la misma cantidad de refuerzo que el diseño por DES; por ejemplo, la relación entre la cantidad de refuerzo para el diseño con DMO y el diseño con DES es 1.1 y 1.0 para los casos de mínima deriva y de máxima deriva, respectivamente.

En el edificio 12P localizado en Barranquilla (figura 9a), es evidente que la cantidad total de refuerzo requerido para el diseño con DMI es menor que para los diseños con DMO y DES. Adicionalmente, el diseño con DES requiere menor cantidad de refuerzo que el diseño con DMO. La relación entre el total de refuerzo que se requirió para DMO y para DMI es de 2.1 para los dos casos de mínima deriva y máxima deriva, respectivamente. Para Bogotá (figura 9b) se observa que, cuando el sistema se acerca a la deriva máxima permisible, el diseño con DMO requiere menor cantidad de refuerzo total que el diseño con DES; cuando se aleja de esta deriva, las cantidades entre ambos diseños son prácticamente las mismas; por ejemplo, la relación entre el total de material que se requiere para DMO y para DES es 1.0 y 1.1, para caso de mínima deriva y máxima deriva, respectivamente.

\section{CONCLUSIONES}

En este estudio se ha evaluado y discutido la variación de las cantidades de refuerzo en dos edificios con sistema estructural de muros y losa. El estudio incluyó un edificio de baja altura de 5 pisos y uno de mediana altura de 12 pisos. Estos edificios se proyectaron en zonas extremas de amenaza sísmica extremas; es decir, una zona de amenaza sísmica baja y zona de amenaza sísmica intermedia. En el estudio se evaluaron dos opciones en cada edificio: que el sistema se acercara a la deriva máxima permisible, o que el sistema se alejara de esta deriva máxima. Este es el intervalo de desplazamientos en que un sistema estructural podría desempeñarse. Los resultados obtenidos de este estudio permitieron generar algunos criterios para el planteamiento de la solución estructural en proyectos de vivienda construidos con sistemas industrializados de muros en concreto reforzado.

En el edificio de 5 pisos (alrededor de $12 \mathrm{~m}$ de altura), la cantidad de refuerzo está gobernada por el refuerzo en el alma. En estos edificios de baja altura se puede esperar un incremento de acero de refuerzo que varía entre $10 \%$ y $20 \%$ en un diseño con DES con respecto al diseño con DMI. Adicionalmente, a medida que la edificación se acerca a la deriva máxima permisible, menor es el incremento del acero. Se debe tener en cuenta que la instalación de los elementos especiales de borde, que no se requieren en DMI y sí se requieren en DES y en DMO, pueden generar costos administrativos 
extras en tiempo, debido a que se requiere mayor atención por la aparición de elementos especiales de borde.

En el edificio de 12 pisos (alrededor de $30 \mathrm{~m}$ ), el protagonismo en las cantidades de refuerzo lo pierde el refuerzo en el alma, y empieza a lograr mayor participación el refuerzo requerido para los elementos de borde. Estas tendencias están directamente relacionados con el modo de comportamiento de edificios de baja altura (por cortante) y mediana altura (por flexión). En estos edificios de mediana altura localizados en zona de amenaza sísmica baja, cuando se realiza el diseño con DMO o DES en lugar de DMI se podría llegar a requerir dos veces la cantidad de material de refuerzo que la que se podría requerir para el diseño con DMI. Si el edificio se localiza en zona de amenaza sísmica intermedia, se podría lograr un ahorro de hasta $10 \%$ en la cantidad de refuerzo en los muros con un diseño con DES con respecto al diseño con DMO. Al momento de plantear la solución estructural de estos edificios de mediana altura, se deben considerar algunas características clave que podrían mantener este ahorro; por ejemplo, conservar una relación de densidad de muros $D_{x} / D_{y}$ alrededor de dos, acercar la estructura a la deriva máxima permisible, tener la deriva máxima del sistema superior o igual a $0.9 \%$, y ubicar la mayor cantidad posible de muros dúctiles $\left(H_{w} / L_{w}>3\right)$ en la dirección de menor densidad de muros, pero tratando de conservar la relación de densidades de muros indicada.

Las recomendaciones realizadas para edificios de 5 pisos, $f_{c}^{\prime}=21 \mathrm{MPa}$ podrían aplicar a edificios de menor altura, y las recomendaciones para el edificio de 12 pisos, $f_{c}^{\prime}=28 \mathrm{MPa}$, podrían aplicar para edificios de mayor altura. En el intervalo de 5 a 12 pisos no se puede aplicar ninguna de las recomendaciones obtenidas, pues no se conoce exactamente para cúal altura la tendencia de los resultados deja de ser similar a la obtenida para el edificio de 5 pisos, ni con concreto de diferente resistencia al usado en este trabajo, pues la resistencia del concreto también influye significativamente en el detallado.

\section{AGRADECIMIENTOS}

Los autores expresan su agradecimiento a la Vicerrectoría de Investigaciones de la Universidad Militar Nueva Granada por el apoyo financiero en el Proyectos INGIMP-2130. También se agradece a la empresa MARVAL, especialmente a su Departamento de Diseño Estructural por el aporte de la información con que se desarrolló el estudio. Adicionalmente, los autores agradecen a la Ingeniera María Constanza García Celis por su acompañamiento durante la larga y comprometida tarea de tabular las cantidades de despieces. 


\section{REFERENCIAS BIBLIOGRÁFICAS}

[1] S. L. Uribe et al., Influencia en la variación de la respuesta estructural y cuantías de diseño de una edificación con sistema muros de carga para una zona de amenaza sísmica baja e intermedia al modificar los requisitos mínimos de disipación de energía, presentado en XXIV Congreso Nacional y XIII Congreso Internacional de estudiantes y profesionales de Ingeniería Civil, Bogotá, 2014.

[2] Comisión Asesora Permanente para el Régimen de Construcciones Sismo Resistentes, Reglamento Colombiano de Construcción Sismo Resistente, NSR-10., Ministerio de Ambiente, Vivienda y Desarrollo Territorial, 2010.

[3] Alcaldía Mayor de Bogotá, Microzonificación Sísmica de Bogotá D.C., Decreto No. 523 de 16 Dic. 2010.

[4] F. Mayagoitia et al., Construcción de vivienda con sistemas industrializados de muros en concreto, Bogotá, ASOCRETO, 2010, pp. 109.

[5] J. Carrillo et al., "A review of conceptual transparency in US and Colombian seismic design building codes.” Ingeniería e Investigación, 33(2): pp. 24-29, 2013.

[6] J. C. McCormac y L.K Nelson, Design of Reinforced Concrete., USA, John Wiley \& Sons, Inc; pp. 721, 2006.

[7] J.P Moehle et al., "Seismic design of cast-in-place concrete special structural walls and coupling beams", NEHRP Seismic Design Technical Brief, pp. 33, 2012.

[8] M.A. Hube et al., "Seismic behavior of slender reinforced concrete walls". Engineering Structures, 80: pp. 377-388, 2014.

[9] C. Alarcon et al., "Effect of axial loads in the seismic behavior of reinforced concrete walls with unconfined wall boundaries". Engineering Structures, 73: pp. 13-23, 2014.

[10] L.M Massone y E.E. López, "Modeling of reinforcement global buckling in RC elements", Engineering Structures, 59: pp. 484-494, 2014. 